\title{
OPEN DATA EN APLICACIONES MÓVILES: NUEVOS MODELOS PA- RA LA INFORMACIÓN DE SERVICIO ${ }^{\mathrm{i}}$
}

\section{Open Data in Mobile Applications, New Models for Service Information}

\author{
Dr. Manuel GÉRTRUDIX BARRIO \\ Profesor Titular, Universidad Rey Juan Carlos, España \\ E-mail: manuel.gertrudix@urjc.es \\ (D) http://orcid.org/0000-0002-5869-3116
}

\author{
Dr. Sergio ÁLVAREZ GARCÍA \\ Profesor Visitante Doctor, Universidad Rey Juan Carlos, España \\ E-mail: sergio.alvarez@urjc.es \\ (iD http://orcid.org/0000-0001-9477-2148
}

\author{
Dr. Mario RAJAS FERNÁNDEZ \\ Profesor Contratado Doctor, Universidad Rey Juan Carlos, España \\ E-mail:mario.rajas@urjc.es \\ (iD http://orcid.org/0000-0002-3807-8327
}

Fecha de recepción del artículo: 26/02/2016

Fecha de aceptación definitiva: 03/04/2016

\begin{abstract}
RESUMEN
La conjunción de la apertura de datos por parte de las administraciones públicas y la proliferación de los dispositivos de movilidad favorece la creación de nuevos servicios, la mejora en la prestación de los existentes y la configuración de nuevos usos comunicativos por parte de los ciudadanos. El desarrollo y puesta a disposición de aplicaciones gratuitas que utilizan los datos abiertos, ofreciendo información de utilidad, en tiempo real, adaptada a la experiencia del usuario y/o geolocalizada, plantea una reformulación del concepto de "información de servicio", ya que ofrece tanto a los ciudadanos como al sector infomediario un espacio emergente para el desarrollo de nuevos modelos de producción, diseminación y consumo de este tipo de información. Desde la contextualización teórica de los procesos de sensorización de la realidad, el registro móvil de la experiencia cotidiana, o la reinterpretación de la información de servicio, se analiza el papel de los datos abiertos en el sector público en España y su aplicación concreta en la creación de apps de servicio basadas en estos. Se revela la extraordinaria emergencia de esta nueva forma de acceder a la información de servicio y su utilidad para la toma de decisiones en la vida cotidiana.
\end{abstract}


Palabras clave: datos abiertos; información de servicio; apps; teléfonos inteligentes; movilidad; experiencia de usuario.

\begin{abstract}
The combination of open data generated by government and the proliferation of mobile devices enables the creation of new information services and improved delivery of existing ones. Significantly, it allows citizens access to simple, quick and effective way to information. Free applications that use open data provide useful information in real time, tailored to the user experience and / or geographic location. This changes the concept of "service information". Both the infomediary sector and citizens now have new models of production and dissemination of this type of information. From the theoretical contextualization of aspects such as processes datification of reality, mobile registration of everyday experience, or reinterpretation of the service information, we analyze the role of open data in the public sector in Spain and its application concrete in building apps based on this data sets. The findings indicate that this is a phenomenon that will continue to grow because these applications provide useful and efficient information to decision-making in everyday life.
\end{abstract}

Keywords: Open data; Service information; Apps; Smartphone; Mobility; User experience.

\title{
1. INTRODUCCIÓN: LA SENSORIZACIÓN DE LA REALIDAD
}

La plena implantación de los dispositivos móviles ha producido cambios esenciales en las formas de comunicarnos, de acceder a la información, de crearla o compartirla. A nivel mundial, los últimos datos del Informe Ditrendia (2015) contabilizan más de 7.300 millones de teléfonos móviles, y un promedio de tiempo de uso de 177 minutos, superando ya el de la televisión (168 minutos). En España, según el Informe de Indicadores destacados de la Sociedad de la Información (ONTSI, 2016) el número de líneas de telefonía móvil alcanza los 50,66 millones, lo que supone una penetración del 109,1\%, es decir, que hay más líneas activas que habitantes. De este modo es, de forma destacada, el país con un mayor número de smartphones asociados a las líneas móviles, un 87\% (Ditrendia, 2015, p. 4).

De las múltiples dimensiones en las que impactan estos cambios, este texto reflexiona sobre cómo se está viendo modificada la denominada información de servicio. En este contexto de democratización de los recursos tecnológicos, de automatización de procesos informáticos, y en los que los móviles son tanto sensores como pantallas en abismo, se produce un profundo replanteamiento de los flujos de generación, distribución y consumo del ámbito de la información de servicio. Aquella información ligada a los aspectos más cotidianos de la vida de los lectores-usuarios y a la que dieron cabida los medios de comunicación de masas especialmente a partir de los años 80 para ofrecer información de utilidad (Knight, 1999), si bien la vinculación entre servicio y periodismo está largamente acreditada desde sus orígenes (Diezhandino, 1993). La información que cubre aspectos como el tiempo, la salud, el medio ambiente, la alimentación, o los viajes ha pasado de estar gestionada a través de los procesos convencionales de los grandes medios, en la lógica del periodismo "doméstico", a sistemas basados en la gestión de grandes cantidades de datos estructurados que son puestos a disposición y representados directamente a través de aplicaciones para smartphones y tablets, widgets o gadgets para sitios web o aplicaciones de escritorio.

Los profesionales que exploran los nuevos caminos de la práctica comunicativa en el entorno digital ponen de manifiesto la necesidad de actualizar los enfoques y las prácticas de los medios para que la ciudadanía pueda obtener un beneficio real de este fenómeno. De ahí el interés que ofrece analizar cómo el desarrollo tecnológico está impeliendo a hacerlo.

En esta línea, el texto realiza una aproximación descriptiva al fenómeno de los datos abiertos en aplicaciones móviles, explorando los nuevos modelos que esta disponibilidad genera para el desarrollo 
de productos dirigidos a satisfacer la necesidad de la información de servicio. Se parte del presupuesto de que la disponibilidad inmediata y actualizada de información de utilidad ajustada a los intereses y necesidades específicas del sujeto individualiza su consumo ofreciendo, a través de dispositivos de propósito general como los smartphones o tablets, experiencias de usuario adaptadas y enriquecidas que transcienden y reformulan el concepto convencional de la información de servicio.

Para evaluar esta propuesta se ha tomado como caso de análisis las apps publicadas en el portal datos.gob.es que reutilizan datos abiertos de la administración pública española. Considerando la taxonomía del catálogo de datos.gob.es, que establece 22 categorías tomadas de otras clasificaciones de referencia como EUGO, INE o EUROSTAT (Secretaría de Estado para la Función Pública, 2014), se ha realizado un análisis descriptivo de una muestra de conveniencia de apps de las cinco categorías que cuentan en datos.gob.es con un mayor número de accesos y descargas. El análisis repara en el tipo de información que facilita la app, la finalidad y función para la que fue realizada, y la utilidad que, en términos de información de servicio, ofrece.

\section{LOS MÓVILES EN EL REGISTRO DE NUESTRA EXPERIENCIA COTIDIANA}

Los antecedentes etimológicos de "registrar" conectan con los términos latinos regestum y regesta. El primero es el dato inscrito; el segundo, el soporte sobre el que se asentaba cada regestum relevante para la administración y que estaba relacionado con las personas o las actividades sociales y económicas. Nos traslada, pues, a la idea de reiteración, de anotación sistemática de información con el objeto de poder ser recuperada para ofrecer una utilidad, para proveer un "servicio".

Desde esos orígenes, las capacidades de registro del ser humano no han parado de evolucionar, de hacerse más complejas, metódicas y poderosas. En su extenso recorrido, que tan minuciosamente ha sido descrito por autores como Briggs \& Burke (2002), los dispositivos móviles representan un estadio más, pero, sin duda, un paso de transcendentes consecuencias por mor de la extraordinaria potencia de procesamiento, la capacidad de contabilizar, inscribir y propagar, en tiempo real, no solo información multimodal, sino datos estructurados que pueden ser captados, tratados y analizados sistemática y sincrónicamente.

Estas capacidades proponen singulares oportunidades en aspectos como la personalización de la experiencia de usuario. Sin duda, resulta sugerente que la datificación de la actividad del sujeto, el análisis comportamental de sus hábitos a partir de la discretización de estos en datos estructurados, facilite experiencias adaptativas, basadas en contenidos inteligentes -smart content- (Saffari, Maertens, \& Miazzo, 2010).

Pero no faltan análisis críticos y distópicos. Briggs \& Burke advertían ya hace más de una década: "hay críticos que temen que internet socave todas las formas de 'autoridad', afecte de manera adversa a la conducta y ponga en peligro la seguridad individual y colectiva” (2002, p. 15). Y es que, como sucede en tantos otros aspectos, la situación actual nos propone interesantes contradicciones que expresan la complejidad de la realidad en la que vivimos.

De alguna forma, esta antilogía, actualiza la Paradoja de Jevons en una sociedad alimentada por la cultura del consumo en la que el abaratamiento de los costes generados por el perfeccionamiento tecnológico y la eficiencia productiva acaba extendiendo el uso de los productos tecnológicos asociados, en nuestro caso de estudio el móvil, lo que acaba derivando dentro de una compleja cadena en un consumo mayor global (Lazzarini \& Cendra, 2009). De este modo, podemos comprender la extraordinaria rapidez y la enorme implantación que el uso de los smartphones ha alcanzado en tan solo 
un lustro, hasta el punto de que muchos ciudadanos disponen de más de una línea activa simultáneamente como se ha referido ya.

Por otra parte, a pesar de la importancia que damos al control de nuestra privacidad, lo cierto es que el móvil se ha convertido en un instrumento ubicuo, una suerte de prótesis multifuncional, reinterpretando a Trejo (2006), en la que la función no solo transforma el órgano sino que se osmotiza con él hasta configurar su comportamiento. En esa integración ad infinitum, el placer de la extensibilidad que nos otorga, de poder, de pseudocontrol, atenúa las reservas del análisis crítico y la prevención ante todas aquellas otras funciones paralelas, a veces semiocultas, del dispositivo.

Por ejemplo, cada vez son más habituales las aplicaciones que registran o trazan la posición GPS del móvil o su actividad con consentimiento del usuario que las instala -como Cell Tracker o MSPY-, recopilando todo tipo de información asociada al dispositivo como el contenido registrado (imágenes, vídeos, actividad en las aplicaciones...), o el comportamiento del usuario (pulsación de teclas, monitorización de la línea telefónica o de las comunicaciones de datos...) (Guillén, 2016) (OSI, 2016).

No obstante, es cierto que la aparición de los conocidos casos de vigilancia de las comunicaciones como los realizados por la NSA, la aparición mediática recurrente de informaciones sobre acceso a datos o contenidos en dispositivos móviles (Cantón, 2016) mediante todo tipo de técnicas como canales encubiertos (Bilic, 2016), y las actuaciones e iniciativas como el OSI (Oficina de Seguridad del Internauta) del INCIBE (Instituto Nacional de Ciberseguridad) (2016) han generado una cierta prevención por parte de los usuarios, y una mejora de los conocimientos sobre la protección de estos dispositivos. Cierto que "cada vez más las personas y las empresas son más conscientes de las repercusiones que pueden tener sobre ellas el robo o divulgación de sus datos privados, y por eso crece también el interés por conocer los hechos relevantes en este ámbito" (Cantón, 2016, 3.1). Pero aún se está lejos de una auténtica cultura de ciberseguridad y de protección de la privacidad en estos entornos, como así vienen refiriendo distintos estudios en los últimos años (Pérez San José, 2012) que ponen de manifiesto, además, la constante tensión entre nuevas medidas de seguridad de los dispositivos y las amenazas emergentes (Thomas, Beresford, \& Rice, 2015)

Todo ello sin contar con los riegos derivados de las denominadas "amenazas humanas", como las que se derivan de la pérdida del smartphone. Como han demostrado los experimentos sociotecnológicos de las diferentes ediciones del proyecto "Honey Stick" (Symantec, 2012), estas representan una verdadera amenaza en términos de seguridad, pues, en el caso de México, el 97\% de los equipos extraviados sufireron al menos un intento de acceso a aplicaciones y archivos, y en un 90\% de los casos se accedió a datos y aplicaciones personales (Symantec, 2014).

Una discusión, en todo caso, que se prolongará de forma mutante, tanto como lo hagan las tecnologías que extiendan, aún más, oportunidades y amenazas, fortalezas y debilidades. En las fronteras de la piel electrónica (El País, 2011) o de los humanoides micro-robots de compañía que, se anticipa, sustituirán a los smartphones (El País One, 2016), el debate está servido.

\section{REINTERPRETANDO LA INFORMACIÓN DE SERVICIO}

La información de servicio posee ese valor de utilidad que va más allá de los temas sociales, políticos o económicos que han determinado la agenda temática de los medios habitualmente (Diezhandino, 1994, p. 66). Ha sido definida como: 
aquella información, ofrecida oportunamente que pretende ser del interés personal del lector-oyente-espectador; que no se limita a informar sobre sino para; que se impone la exigencia de ser útil en la vida personal del receptor, psicológica o materialmente, mediata o inmediatamente, cualquiera que sea el grado y el alcance de esa utilidad. La información cuya meta deja de ser ofrecer datos circunscritos al acontecimiento, para ofrecer respuestas y orientaciones. (Diezhandino, 1994, p. 89).

Esa información orientada a la acción se ha visto reinventada por la disponibilidad de recursos inmediatos. Recursos que proveen soluciones informativas que, de forma ágil y directa, facilitan la toma de decisiones de los usuarios para ámbitos que se han consolidado en la sociedad del bienestar, y que afectan a cuestiones cotidianas, de relativa relevancia, pero cuya disponibilidad resulta esencial para la percepción de una satisfacción psicológica de los sujetos. No podemos obviar, en este sentido, el cambio que se ha producido en los modelos de acceso y consumo de información en poco tiempo, si consideramos las dificultades de convergencia que reflejaban algunos estudios hace tan solo unos años (Ruiz del Olmo, 2012). Superada esta situación, actualmente "tres de cada diez personas que tienen un Smartphone miran su teléfono móvil más de 50 veces al día” (Ditrendia, 2015, p. 26), y que el nivel de "dependencia" que se ha establecido con los hábitos de consumo móvil son extraordinarios, generando un continuo de conectividad que es aún más significativo en el caso generaciones como millennials, los nativos digitales, pero también de todo el "vasto grupo poblacional relacionado estrechamente con las TIC y que ha hecho de las redes un espacio social y personal básico de su actividad, de su forma de habitar y relacionase con el mundo" (García \& Gertrudix, 2012, p. 7). Sin duda, se reivindica esa orientación del periodismo de servicio que "más que al ciudadano atiende al individuo" para satisfacer necesidades concretas y "ofrecer o sugerir, implícita o explícitamente, alternativas, propuestas, soluciones, elementos, en fin, útiles, para mejor afrontar la vida cotidiana" (Diezhandino, 1993, p. 123). Algunos autores observan, no obstante, un alcance híbrido del sujeto social, entendido simultáneamente como ciudadano, como consumidor y como cliente (Knight, 1999, p. 525), y otros ponen de relieve el cambio que venía produciéndose ya en la conceptualización del periodismo de servicio, como periodismo utilitario -"how-to-do"-, a un "espíritu de servicio" por parte de los redactores (Bala Aggarwal, 2006).

En un paso más allá, la apertura de fuentes de datos suministra la creación de sistemas de comunicación permanente entre sistemas. Pero también entre sistemas, dispositivos y usuarios, de manera que no solo se utilizan datos ya existentes, sino que se recolectan y diseminan nuevos datos que contribuyen a mejorar la experiencia global del usuario con el servicio, proveyendo información totalmente actualizada y adaptada en función de las necesidades concretas del ciudadano en ese momento.

Pero además, los dispositivos móviles se suman a una red de receptores y sensores de datos que, de forma automática o mediante pequeñas acciones de los usuarios, contribuyen a generar nuevos datos; datos que permiten a los gestores disponer de una mayor cantidad de información para la gestión de los propios servicios, como, por ejemplo, los flujos urbanos, los niveles de polución, etc.

Las capacidades tecnológicas de los smartphones, las funcionalidades de geolocalización, ubicuidad de acceso y los modelos de interacción con otros contenidos, abastecen la creación de capas de servicio informativo de alto valor añadido. Las funcionalidades condesadas, altamente selectivas y especializadas que proveen las apps, unidas al aprovechamiento que hacen de las cada vez mayores capacidades de procesamiento de los teléfonos inteligentes, ofrecen a los usuarios una extraordinaria posibilidad de personalización de la experiencia; ello ha contribuido a la auténtica explosión del fenómeno. El informe Ditrendia ofrece algunos datos esenciales para comprenderlo: un $90 \%$ del tiempo de exposi- 
ción al móvil se produce ya en el uso de apps. Y ello en un mercado que crece a razón de 40.000 nuevas aplicaciones mensuales, para un inventario global, si sumamos Google Play Store y Apple Store, de más de 2 millones de aplicaciones (2015, p. 5). Interpretados estos datos desde la reconsideración de la información de servicio, muchas apps han logrado el sueño buscado por los escritores de periodismo de servicio que, en palabras de Jessica Lockhart revela: "writers' aspirations aside, service is what hooks readers - providing tips for doing things faster, easier and smarter-and keeps them coming back for more” (2008, 5.1). Los usuarios logran solucionar sus necesidades más rápido, de forma más fácil, sencilla y óptima, y la satisfacción del resultado estimula y refuerza el hábito de uso.

El informe de Mobile Marketing (IAB Spain, 2015) clasifica las actividades realizadas con el móvil en: sociales, lúdicas, consultivas y funcionales. De estas, son las consultivas (consultar el tiempo, cuestiones de salud o bienestar, viajes, restauración...) y las funcionales (utilidades) las que se relacionan de forma más inmediata con la información de servicio. En este último informe, los usuarios declaran que el uso de las mismas es fundamentalmente semanal, frente a las sociales y a las lúdicas, cuyo uso habitual es a diario.

En términos lamarckianos, el cambio de medio, el ecosistema y el hábitat tecnológico, y, en su adaptación, el organismo (los usuarios de información) se acomodan (generan nuevas prótesis tecnológicas) para satisfacer las nuevas necesidades de manera más perfecta: la función crea el órgano y la necesidad la función. Desde el análisis de la dieta de medios, la modificación del modelo de consumo de la información de servicio producido por esa integración automatizada de datos en tiempo real y acceso adaptado a través de las apps en dispositivos móviles, unido a las opciones que estos permiten a los ciudadanos para configurar sus propio relato informativo, extiende indudablemente el concepto de dieta mediática y repertorio de medios (García, Gértrudix \& Gértrudix, 2014). Concepto definido por Hasebrink y Domeyer como "el resultado de múltiples situaciones y comportamientos individuales de selección $[. .$.$] que configuran una estructura individual de consumo en la vida diaria. Los repertorios$ de medios están compuestos por diferentes contactos con los medios, incluyendo una variedad de diferentes medios y contenidos" (2012, p. 759).

Ello actualiza la reflexión que realizaba Philip Meyer ya en 1993 sobre la necesidad de facilitar al lector medios eficaces para extraer información de los relatos periodísticos (1993, p. 32), y cobra todo su sentido considerando la dificultad que existe actualmente para focalizar la atención ante la plétora de estímulos que reciben los sujetos a través de distintos canales de forma simultánea. Estímulos que se solapan de forma continua e iterativa generando condiciones como la distracción (Klingberg, 2010), la fatiga informativa (Lewis, 1996) o la lectura fragmentada (Carr, 2010), y que ya fueron anticipados por la teoría de la Economía de la Atención por Simon (1971) o Goldhaber (1997).

Estos cambios en los modelos de información de servicio también han sido estudiados desde otras dimensiones como el impacto de la convergencia entre redes sociales y dispositivos móviles (González, 2012), el valor que el flujo de los medios sociales otorgan a la información de servicio (Hahn, 2013), la co-creación informativa basada en la participación y en la creación de comunidades favorecida por la tecnología de la web social (Usher, 2012), la perspectiva de los profesionales ante este cambio (Yousuf Ali \& Kaur, 2015) o la personalización basada en la configuración de la información mediante la contextualización de proximidad gracias a la localización mediante GPS (Oppegaard \& Rabby, 2015). González Molina (2012) señala la ubicuidad, la personalización y la participación de los usuarios que facilitan los dispositivos móviles como elementos clave de este cambio, y Franco y Pellicer destacan cómo internet ha desplazado la centralidad que detentaban los medios hasta hace poco, "potenciando los mercados de nichos y las pequeñas comunidades" (2014, pp. 127-128). 


\section{El PAPEL DE LOS DATOS ABIERTOS DEL SECTOR PÚBLICO EN ESPAÑA}

Los datos abiertos vinculados son datos brutos o iniciales estructurados publicados en la Red que son interoperables y, por lo tanto, pueden interconectarse entre sí para ofrecer una mejor experiencia de usuario. Como indican Bizer, Cyganiak y HeathTom, "the goal of Linked Data is to enable people to share structured data on the Web as easily as they can share documents today" (2011,1.1). Para ello es necesario que cumplan determinados requisitos: a) estar publicados bajo un modelo de licencia abierta, b) los enlaces vinculantes de los datos deben estar representados de la forma más simple utilizando RDF (Resource Description Framework), c) deben de tener un localizador único (URI), d) y estar publicado en línea bajo el protocolo http. Ello facilita el intercambio y la explotación de estos datos, y, lo que es aún más relevante, su reutilización de forma creativa de forma que cualquier usuario pueda generar nuevos productos informativos que aporten valor añadido a la disponibilidad de esos datos.

La Directiva 2003/98 relativa a la reutilización de la información del sector público marcó un paso importante hacia un proceso de impulso de la puesta a disposición de los datos públicos y de armonización de las normativas y sistemas a nivel europeo con la finalidad de garantizar modelos compartidos para la explotación de la información en condiciones equitativas, proporcionadas y no discriminatorias. A pesar de que no establecía a los estados miembros la obligatoriedad de autorizar la reutilización de documentos, sí instaba a estos de forma directa para facilitar el acceso a los mismos, definiendo documento de una forma flexible y amplia: "abarca todas las formas de representación de actos, hechos o información, y cualquier recopilación de los mismos, independientemente del soporte (escrito en papel, almacenado en forma electrónica o como grabación sonora, visual o audiovisual), conservados por los organismos del sector público" (Parlamento Europeo, 2003, 11).

La importancia gradual del fenómeno, en plena expansión aún, muestra que en el ámbito sectorial el crecimiento del sector vendrá dado por la creación de nuevas empresas y la reformulación de algunas del ámbito informativo hacia el sector infomediario, entendido este como el compuesto por aquellas "empresas que generan aplicaciones, productos y/o servicios de valor añadido destinados a terceros, a partir de la información del Sector Público" (Dekkes, Polman, Velde, \& Vries, 2006, p. 3). A la luz del creciente número de iniciativas, es indudable la emergencia, interés y oportunidad que representa, pero también la experiencia de puesta en marcha de las iniciativas necesarias no está exenta de dificultades: "no es tan sencillo como publicar ciertos datos a través de una web y esperar que los beneficios se materialicen de forma inmediata" (RED.es, 2014, p. 1).

En España, el instrumento utilizado por el Ministerio de Industria, Energía y Turismo -a través de RED.es- para promover la "cultura de reutilización de la información en el ámbito de la Administración Pública" ha sido el Proyecto Aporta, iniciado en el año 2009, y cuyas principales actuaciones son: la creación y soporte del portal aporta.es, el impulso del catálogo de datos abiertos data.gob.es, y las actuaciones de concienciación, difusión y estímulo de la cultura de datos abiertos y reutilización de información (Red.es, 2009). No obstante, el número de iniciativas de datos públicos abiertos ha aumentado de forma notable en los últimos años hasta alcanzar 31 portales, tal como ha sido recogido por proyectos como el Mapa actual de las iniciativas Open Data en España (Meijueiro, 2014), el Mapa Mundial de Catálogos de Datos Públicos (CTIC, 2014) o el Informe de la Fundación Orange sobre los Datos abiertos en las Comunidades Autónomas y los mayores ayuntamientos (2014). Esto se refleja también en iniciativas como el Global Open Data Index que sitúa a España en 2015 en el puesto 17 a nivel internacional, con una subida de 14 puestos en relación a 2014, y un 55\% de valoración de apertura (Open Knowledge Network, 2015). Esta progresion se viene apuntando desde hace algunos años (Simón et al., 2012), si bien no está exenta de algunas críticas que, como los análisis realizados por la Fundación Civio (Cabo, 2013) ponen de relieve la dificultad de sostenibilidad en el mantenimiento 
de los portales de datos abiertos, la falta de metodologías comunes que faciliten la interoperabilidad o el retroceso que supone la comercialización de algunos datos, como los meterológicos, que hasta hace poco se ofrecían de forma gratuita.

\section{CASOS DE INFORMACIÓN DE SERVICIO EN LAS APPS BASADAS EN DA- TOS ABIERTOS}

Como se ha tratado, la lógica de la cultura mashup "ha propiciado el desembarco de una nueva generación de aplicativos que mixturan propiedades de varias herramientas para crear o configurar nuevas soluciones mediante asociaciones flexibles (loosely coupled) (García \& Gértrudix, 2011, p. 126). En el caso de las apps basadas en datos abiertos se aprovecha el acceso a dos o más fuentes para generar valor añadido mediante la combinación de informaciones que, hasta ese momento, operaban por separado. Un ejemplo muy habitual suele ser la composición de datos GIS (Sistemas de información geográfica) con los derivados de cuestiones como el transporte, la vivienda o la salud, pero se trata de un proceso vivo, en constante evolución y con una extraordinaria capacidad de innovar en nuevas soluciones informativas.

Sin duda este tipo de servicios que están desarrollando un uso optimizado de los datos abiertos se verá incrementado exponencialmente. No en vano, el número de aplicaciones para dispositivos móviles de esta naturaleza ha crecido de forma extraordinaria en los últimos años, y es una tendencia que no dejará de crecer.

Siete años después de su puesta en marcha, el catálogo de aplicaciones del portal datos.gob.es registra en febrero de 2016 un inventario de apps y desarrollos para web móvil y sistemas operativos como Android o iOS, que se reparten temáticamente de forma muy heterogénea, estando dirigidas a usos potenciales muy diversos. Como se revela en el portal,

los productos y servicios más populares son los derivados de la reutilización de información del ámbito empresarial-económico; geográficocartográfico; jurídico-legal; de transportes; sociodemográfico-estadístico; y meteorológico, entre otros. (Red.es, 2014).

A la hora de clasificar la información de servicio desde esta perspectiva resulta de utilidad la clasificación por sectores temáticos concretada en la Taxonomía del catálogo de datos.gob.es, que establece 22 categorías, desde la Ciencia y la Tecnología hasta la Vivienda, y está basada en otras clasificaciones de referencia como EUGO, INE o EUROSTAT (Secretaría de Estado para la Función Pública, 2014). Para el análisis descriptivo se ha realizado una selección de algunas de las apps con más accesos y descargas desde el portal data.gob.es.

Tabla 1. Catálogo de aplicaciones del portal data-gob.es

\begin{tabular}{|c|c|}
\hline Categoría & Número \\
\hline Medio Ambiente & 1487 \\
\hline Transporte & 37 \\
\hline Urbanismo e infraestructuras & 29 \\
\hline Sector público & 25 \\
\hline Economía & 11 \\
\hline Sociedad y bienestar & 9 \\
\hline Energía & 8 \\
\hline
\end{tabular}

(C) Ediciones Universidad de Salamanca / CC BY - NC ND $\quad$ Fonseca, Journal of Communication, n. 12, 2016, pp. 117-131 


\begin{tabular}{|c|l|}
\hline Ciencia y tecnología & 7 \\
\hline Comercio & 6 \\
\hline Salud & 5 \\
\hline Cultura y ocio & 5 \\
\hline Turismo & 4 \\
\hline Vivienda & 3 \\
\hline Industria & 2 \\
\hline Educación & 2 \\
\hline Legislación y Justicia & 2 \\
\hline Empleo & 2 \\
\hline Demografía & 1 \\
\hline Hacienda & 1 \\
\hline Seguridad & 0 \\
\hline Deporte & 0 \\
\hline Medio Rural & 0 \\
\hline Fut Elaborán propia
\end{tabular}

Fuente: Elaboración propia

a partir de datos de data.gob.es.

Las aplicaciones relacionadas con el medio ambiente son con diferencia las más numerosas actualmente. Dentro de estas se encuentran las destinadas a favorecer conductas comprometidas (reciclaje, conservación, protección...) o las que informan sobre el estado y la calidad del entorno o los niveles de contaminación (agua, aire...).

Por ejemplo, Dónde reciclo es una aplicación que facilita información sobre los puntos de reciclaje en la ciudad de Málaga para todo tipo de residuos (aceite, envases, industria, papel, pilas, ropa, vidrio...) permitiendo a los ciudadanos conocer cuáles están más próximos mediante un buscador o mediante la localización por GPS (Forma masiva, 2015) Aguas y mapas, desarrollada por ESRI, muestra la situación del agua embalsada, el consumo por regiones, personas o actividades económicas. Aire Madrid o Pollen in the air informan sobre la calidad del aire, y Emisiones y Residuos de Complejos Industriales permite comparar las emisiones y residuos de zonas industriales o localizar aquellas que se encuentran más próximas y que, por lo tanto, pueden afectar al ciudadano de forma más directa.

En el caso de la información sobre la previsión del tiempo algunos estudios revelan la importancia de estas apps, ya que, en muchos casos son servicios integrados por defecto en los móviles y es el medio desde el que se consulta, casi en exclusividad, esta información (Ditrendia, 2015, p. 36). La apertura de datos de la AEMET (Agencia Estatal de Meteorología) a través de su catálogo público en la web (AEMET, 2016) facilita una amplia lista de datasets sobre aspectos como los valores climáticos normales, valores extermos absolutos, radiación solar, predicciones del tiempo o escenarios de cambio climático, que son utilizados por numerosas empresas del sector Infomediario para crear aplicaciones con elementos de valor añadido, que proveen a los usuarios de ligeros y útiles sistemas de información metereológica, cuyo uso incide decididamente en las decisiones cotidicanas de los ciudadanos.

Otro ámbito son las apps que entregan información de servicio entorno al transporte y los sistemas de movilidad, con la idea de hacerlos más sostenibles y eficaces, tanto en el ámbito del transporte público con el privado. La importancia e impacto del open data es reconocido como un elemento esencial para el desarrollo futuro de una movilidad sostenible en las ciudades inteligentes 


\begin{abstract}
Open data enhances the value of information services by allowing developers to access a greater number and range of useful information components, without major start-up costs. Many information services are, in turn, freely available either in whole - where finance is provided by investors or advertisers - or to a specified level of service, with enhanced service chargeable by user subscriptions. Thus, economic value is created for the developer. Ultimately, developers may sell shares in their business to generate additional revenue, or otherwise sell the whole company. (The Climate Group, 2014, p. 31).
\end{abstract}

Las aplicaciones del sector transporte combinan habitualmente información geográfica con datos obtenidos de diferentes bancos de datos para permitir conocer en tiempo real la situación de aspectos como el tráfico, los problemas o dificultades que puedan acontecer y cómo sortearlos. Es habitual, asimismo, que los propios usuarios puedan incoporar datos sobre situaciones o imprevistos que estén sucediendo, como un accidente o una obra no señalizada, que no está recogida en el sistema pero que es dada de alta por aquellos que están en ese momento cerca del acontecimiento, lo que contribuye al mismo tiempo a mejorar la información que la administración posee sobre los problemas que afectan a los ciudadanos y, con ello, poder solucionarlos. Los móviles, a través de la acción de sus propietarios, se convierten en sensores activos de una ciudad, carretera o entorno físico que se retroalimenta constantemente.

Algunas aplicaciones de este tipo son Mobility 4 All, orientada a mejorar la mobilidad urbana, Seetys Transporte, que aprovechando la especificación GTFS (feeds de transporte público) muestra la situación en tiempo real del transporte público de ciudades como Madrid, Barcelona o Vitoria Gazteiz, Madrid Metro, Bus y Cercanias o Citymapper, con funcionalidades similares como widgets de tiempo de espera en parada, distancia entre los autobuses y convoyes o rutas y trayectos óptimos, y en algunos casos incluyendo simluación de los desplazamientos de los vehículos que ofrecen el servicio (Seetys, simulador de vehiculos). En otros casos, las apps ofrecen información del estado actualizado de servicios como las redes de bicicletas de alquiler en aquellas ciudades que ofrecen el servicio (Bizi Zaragoza, Málaga Bici...).

Los servicios comparativos también han empezado a tener interesantes propuestas, como Las gasolineras más baratas que, en versión web y web móvil, contextualiza geográficamente datos del Ministerio de Industria, Turismo y Comercio para mostrar cuáles son los surtidores más económicos desde una posición dada, segmentando la información por tipo de carburante o radio de distancia máximo.

Otro bloque importante de aplicaciones están relacionadas con el ámbito de la salud desde diferentes aproximaciones: medicamentos, productos fitosanitarios, información sobre Centros de Salud, Consultorios Locales u Hospitales.

Por ejemplo, la app aempsCIMA, creada por la Agencia Española de Medicamentos y Productos Sanitarios (AEMPS), busca "proporcionar puntualmente a los ciudadanos y profesionales toda la información sobre los medicamentos de forma comprensible para conseguir de esta forma su correcta utilización" (AEMPS, 2014). Para ello ofrece, a través de la app, acceso a las fuentes primaria de información sobre el catálogo de medicamentos de forma actualizada, incluyendo datos de más de 15.000 medicamentos y 1.600 principios activos, que pueden ser recuperados mediante selectores como el nombre, el laboratorio, el principio activo o el grupo terapéutico al que pertenece.

La categoría Sociedad y bienestar recoge aplicaciones cuyos objetivos abarcan desde simplificar la realización de trámites administrativos o actividades (Buscador de trámites de la Administración Pública, 
Mi Pueblo, Papelea), la mejora de la habitabilidad urbana mediante la combinación de datos de los portales urbanos de con los generados por los usuarios al actuar como sensores desde sus apps móviles (Málaga City Sense), o información dirigida a la toma de decisiones sobre cuestiones como el mejor lugar para vivir dentro de una región o una ciudad en función de múltiples criterios como la diversidad cultural o el nivel de vida contextualizados geográficamente (Illustreets), o los espacios adaptados y accesibiles para personas con diversidad funcional (Sin barrera).

Es interesante el apoyo que algunas instituciones internacionales, como la Knight Foundation, están prestando a la innovación para proyectos que tratan de expandir estas posibilidades actuales. En el última edición del Knight Propotype Found de agosto de 2015, el proyecto Placelet -aún en desarrolloplantea el diseño de un sistema para colectar, analizar y visualizar datos de la experiencia humana en las plazas urbanas. Se trata de un modelo de sensorización de ámbitos urbanos que integra los datos generados por los usuarios con el registro aspectos como el movimiento, el sonido o la calidad del aire, a través de una red de sensores físicos, con el objetivo de mejorar las condiciones de vida en los ámbitos urbanos (Knight Foundation, 2015).

Las apps encuadradas dentro de Sector Público recogen soluciones en muchos casos multifuncionales con servicios integrados de comunicación, adaptación de información por proximidad mediante geolocalización por GPS, trámites, notificación de emergencias o sistemas de avisos con fitro de geolocalización. Algunas Comunidades Autónomas y grandes ciudades han desarrollado este tipo de servicios entre los que se encuentran diversas aplicaciones del catálogo de servicios móviles de la Generalitat de Catalunya, como la Aplicación gencat que integra servicios de Protección Civil, Tráfico, Tiempo o equipamientos urbanos (Generalitat de Catalunya, 2015), AppValencia, o Aragopedia. En otros casos, como Sielocal 2.0 (2012), concentran una amplísima información sobre la situación económica de la administración municipal facilitando informes automatizados, en tiempo real, sobre numerosos indicadores presupuestarios y contables del ámbito local.

\section{CONCLUSIONES}

La sensorización o datificación de nuestro entorno vital está contribuyendo de forma decisiva a anudar, en una suerte de continuo, las realidades física y virtual. El último impulsor de este proceso están siendo los dispositivos móviles, especialmente los smartphones y las tablets, y de, forma emergente, los wereables y el Internet de las Cosas (IoT), "gracias a los millones de sensores que inundan nuestro mundo [...] y que nos ofrecen una nueva narrativa, en tiempo real, de las mecánicas urbanas, sociales y económicas" (Fundación Telefónica, 2015 p. 6).

En este contexto de profusión informativa, de abundancia y plétora de datos, de recirculación constante, de registro sistemático de la experiencia cotidiana, la entrega de parte de nuestra privacidad ofrece a cambio experiencias de usuario más precisas, funcionales y optimizadas en el consumo de información de utilidad y servicio.

La apertura de datos de las administraciones públicas, su disponibilidad en catálogos de datos estructurados y estandarizados según los criterios exigidos para los Datos abiertos vinculados han favorecido el desarrollo de soluciones de alto valor añadido creadas por entidades y empresas del sector Infomediario. Dichas soluciones se concretan, en buena medida, en aplicaciones disponibles para los principales sistemas operativos de dispositivos móviles o aplicaciones web, en las que, de forma gratuita, los ciudadanos disponen en su smartphone o tablet de un amplio inventario de soluciones con información de servicio adaptada a elementos dinámicos como el comportamiento o el geoposicioamiento, la contextualización geográfica o la selección y filtrado mediante acciones de búsqueda selectiva. 
Esta creciente disponibilidad de recursos, condiciona y requiere un replanteamiento del concepto y de los procesos de elaboración de "información de servicio". La funcionalidad aportada por estas aplicaciones, y la indudable contribución que seguirán realizando en el futuro para ofrecer experiencias de mayor eficacia en la resolución de consultas y orientaciones prácticas en la vida cotidiana, nos lleva a un nuevo estadio de la información de servicio entendida, de forma extensa, como "servicios de información para la toma de decisiones informadas" de alto valor añadido. Estos nuevos modelos quedan caracterizados por rasgos como la inmediatez informativa, la adaptación al individuo y no tanto al colectivo, la creación de experiencias informativas de usuario enriquecidas y adaptadas al comportamiento, la situación o las circunstancias del usuario, la retroalimentación constante entre los servicios de información basados en datos abiertos y los sistemas de recogida que proveen los sensores de los smartphones, y la creación de registros históricos de actividad que son integrados con las miríadas de registros de otros usuarios para generar patrones que anticipen necesidades y tengan carácter predictivo y proyectivo sobre la información que resulta valiosa, útil y eficaz.

Sin embargo, cabe considerar también, como plantean algunos estudios, que la evolución favorable de este sector de apps estará sujeta a decisiones conjuntas en el marco de los responsables en materia de apertura de datos, sobre algunos de los aspectos que, como los procesos de interoperabilidad (Loutas, Varitimou \& Peristeras, 2013) o la profundización en la cultura de datos (Álvarez, Gértrudix \& Rajas, 2014) en el ámbito de la administración pública, así como del sector profesional de la comunicación a la hora de reinventar sus productos y adaptar los perfiles profesionales y sus competencias a este nuevo entorno tecnológico (Gertrudis, Gértrudix \& Álvarez, 2016).

\section{BiBLIOGRAFÍA}

AEMET. (2016). Catálogo de datos abiertos de la AEMET. Disponible en: http://www.aemet.es/es/datos abiertos/catalogo [Fecha de consulta: 26 de enero de 2016].

AEMPS. (08 de 01 de 2014). La AEMPS presenta una aplicación para dispositivos móviles para acercar la información de los medicamentos a profesionales y ciudadanos. Disponible en: http://goo.gl/EslMP4 [Fecha de consulta: 16 de enero de 2016].

Álvarez, S., Gértrudix, M., Rajas, M. (2014). La construcción colaborativa de bancos de datos abiertos como instrumento de empoderamiento ciudadano. Revista Latina de Comunicación Social, 69, 661-683. doi: 10.4185/RLCS-2014-1029.

Bala Aggarwal, V. (2006). Essentials or Practical Journalism. New Delhi: Concept Publishing Company.

Bilic, D. G. (15 de 1 de 2016). Canales encubiertos en entornos móviles: robo de datos en forma sigilosa. Disponible en: http://goo.gl/hcirmX [Fecha de consulta: 31 de enero de 2016].

Bizer, C., Cyganiak, R., \& HeathTom. (2011). How to Publish Linked Data on the Web. Universidad de Manheim. Disponible en: http://goo.gl/lonZ3i [Fecha de consulta: 22 de enero de 2016].

Briggs, A., \& Burke, P. (2002). De Gutenberg a Internet. Madrid: Taurus.

Cabo, D. (7 de 04 de 2013). Portales opendata como aeropuertos sin aviones. Fundación Civio: Disponible en: http://www.civio.es/2013/04/opendata [Fecha de consulta: 9 de marzo de 2016].

Cantón, D. (28 de 01 de 2016). INCIBE. Titulares de ciberseguridad del 2015. Disponible en: https://goo.gl/o7AUyO [Fecha de consulta: 14 de febrero de 2016].

Carr, N. (2010). The Shallows: What the Internet Is Doing to Our Brains. New York: W.W. Norton \& Company Inc.

CTIC. (2014). Mapa Mundial de Catálogos de Datos Públicos. Open Data @CTIC. Disponible en: http://goo.gl/vT7XoC [Fecha de consulta: 17 de febrero de 2016].

Dekkes, M., Polman, F., Velde, R. t., \& Vries, M. d. (2006). MEPSIR. Measuring European Public Sector Information Resources. Paris: European Commision. 
Diezhandino, M. P. (1993). El “periodismo de servicio”, la utilidad en el discurso periodístico. Anàlisi: quaderns de comunicació $i$ cultura, 15, 117-125.

Diezhandino, M. P. (1994). Periodismo de servicio. Barcelona: Bosch.

Ditrendia. (2015). Informe ditrendia: Mobile en España y en el Mundo 2015. Disponible en: http://goo.gl/ikK32N [Fecha de consulta: 16 de febrero de 2016].

El País One. (21 de 02 de 2016). En el futuro tu smartphone será un pequeño robot metido en tu bolsillo. Disponible en: http://goo.gl/oaGwl1 [Fecha de consulta: 22 de febrero de 2016].

El País. (11 de 08 de 2011). Piel electrónica para medir constantes vitales. El País Sociedad: Disponible en: http://goo.gl/TfT0Ei [Fecha de consulta: 27 de diciembre de 2015].

Franco, M., \& Pellicer, M. (2014). Optimismo para periodistas: claves para entender los nuevos medios de comunicación en la era digital. Editorial UOC.

Forma masiva. (2015). Dónde reciclo. dondereciclo.org.es. Disponible en: http://www.dondereciclo.org.es/malaga.php [Fecha de consulta: 14 de enero de 2016].

Fundación Telefónica. (2015). Big Bang Data. Madrid: Fundación Telefónica.

Fundación Orange (2014). Datos abiertos en las Comunidades Autónomas y sus mayores ayuntamientos 2014. Disponible http://www.proyectosfundacionorange.es/docs/eE2014/Datos Abiertos 2014.pdf [Fecha de consulta: 9 de marzo de 2016].

García, F., \& Gértrudix, M. (2011). Naturaleza y características de los servicios y los contenidos digitales abiertos. CIC Cuadernos de Información y Comunicación, 16, 125-138. doi: 10.5209/rev_CIYC.2011.v16.7

García García, F., \& Gértrudix Barrio, M. (2012). El Mare Nostrum digital: Mito, ideología y realidad de un imaginario sociotécnico. Revista ICONO14. Revista Cientifica de Comunicación y Tecnologias Emergentes, 7(1), 7-30. doi: 10.7195/ri14.v7i1.331

García, F., Gértudrix, M. \& Gértrudix, F. (2014) Análisis de la incidencia de la dieta de servicios digitales en la utilidad y confianza de la información en internet en los jóvenes universitarios, Communication \& Society / Comunicación y Sociedad, 27(1), 59-81.

Generalitat de Catalunya. (2015). gencat.cat. Aplicaciones de la Generalitat. Disponible en: http://www.gencat.cat/mobils/cas/index.htm [Fecha de consulta: 11 de enero de 2016].

Gertrudis, M.C., Gértrudix, M. \& Álvarez, S. (2016). Competencias informativas profesionales y datos abiertos. Retos para el empoderamiento ciudadano y el cambio social [Professional Information Skills and Open Data. Challenges for Citizen Empowerment and Social Change]. Comunicar, 47. doi: 10.3916/C47-2016-04

Goldhaber, M. H. (1997). The Attention Economy and the Net . First Monday, 2(4).

González, S. (2012). Contenidos móviles para la comunicación de servicio 2.0 a partir de las redes sociales. Cuadernos de información, 31, 151-162.

Guillén, B. (11 de 02 de 2016). ¿Llevas un espía en el bolsillo? El País Tecnología: Disponible en: http://goo.gl/eNsezu [Fecha de consulta: 12 de febrero de 2016].

Hahn, N. (2013). What good is Twitter? The value of social media to public service journalism. En E. B. Polis, Eurovision Media Strategy. London: London School of Economics and Political Science.

Hasenbrink, U., \& Domeyer, H. (2012). Media repertoires as patterns of behaviour and as meaningful practices: A multimethod approach to media use in converging media environments. Journal of Audience \& Reception Studies, 9(2), 757-779.

IAB Spain. (29 de 09 de 2015). Estudio anual de Mobile Marketing. Interactive Advertising Bureau. Disponible en: http://goo.gl/AWcL3n [Fecha de consulta: 12 de enero de 2016].

INCIBE. (2016). Oficina de Seguridad del Internauta. OSI: Disponible en: http://www.osi.es/ [Fecha de consulta: 8 de enero de 2016]. 
Klingberg, T. (2010). Training and plasticity of working memory. Trends in Cognitive Sciences , 14(7), 317324.

Knight Foundation. (2015). Knight Prototype Found. Disponible en: http://goo.gl/jishdx [Fecha de consulta: 20 de diciembre de 2015].

Knight, G. (1999). Public/Private Service. Service Journalism and the Problems of Everyday Life. European Journal of Communication, 14(4), 525-547.

Lazzarini, B., \& Cendra, J. (2009). Innovació tecnológica y cultura del consumo: la necesidad de una aproximación sistémica. Congrés UPC Sostenible 2015. Barcelona: Universitat Politécnica de Catalunya.

Lewis, C. A. (1996). Green Nature/Human Nature: The Meaning of Plants in our Lives. Chicago: University of Illinois Press.

Lockhart, J. (2008). 5 Reasons to Love Service Journalism. Review of Journalism. Disponible en: http://rri.ca/5-reasons-to-love-service-journalism [Fecha de consulta: 21 de diciembre de 2015].

Loutas, N., Varitimou, A., \& Peristeras, V. (2013). Unraveling the mystery of Open Government Data Apps. En Workshop on using open data: policy modeling, citizen empowerment, Data Journalism (Vol. 1).

Meijueiro, L. (26 de 03 de 2014). Mapa actual de las iniciativas Open Data en España. Open Data @ CTIC. Disponible en: http://goo.gl/KQ44uc [Fecha de consulta: 14 de febrero de 2016].

Meyer, P. (1993). Periodismo de precisión. Barcelona: Bosch.

MITYC - Plan Avanza2. (2009). Proyecto APORTA. Disponible en: http://www.aporta.es/ [Fecha de consulta: 5 de febrero de 2016].

ONTSI. (01 de 2016). Indicadores destacados de la Sociedad de la Información. Disponible en: http://goo.gl/EFkBoE [Fecha de consulta: 12 de febrero de 2016].

Open Knowledge Network. (2015). Global Open Data Index. Disponible en: http://index.okfn.org/place/spain/ [Fecha de consulta: 8 de marzo de 2016].

Oppegaard, B., \& Rabby, M. (28 de 07 de 2015). Promimity. Revealing new mobile meanings of a traditional news concept. Digital Journalism.

OSI. (05 de 02 de 2016). ¡Mi móvil está poseído! Oficina de Seguridad del Internauta Disponible en: https://goo.gl/hNmbw7 [Fecha de consulta: 14 de febrero de 2016].

Parlamento Europeo. (11 de 2003). DIRECTIVA 2003/98/CE DEL PARLAMENTO EUROPEO Y DEL CONSEJO de 17 de noviembre de 2003 relativa a la reutilización de la información del sector público. Diario Oficial de la Unión Europea. Disponible en: http://goo.gl/9ssDc [Fecha de consulta: 17 de febrero de 2016].

Pérez San José, P. (. (2012). Estudio sobre seguridad en dispositivos móviles y smartphones (1er cuatrimestre 2012). INTECO. León: INTECO.

Red.es. (2014). Datos.gob.es. Portal de datos abiertos de la administración Pública Española. Disponible en: http://goo.gl/nU7Dwo [Fecha de consulta: 18 de febrero de 2016].

Red.es. (2009). Reutilización de la Información de la Administración Pública. Proyecto Aporta Disponible en: http://goo.gl/s3q0El [Fecha de consulta: 2 de febrero de 2016].

RED.es. (2014). Tendencias Actuales en iniciativas Open Data. aporta.es. Madrid: Ministerio de Industria, Energía y Turismo.

Ritzer, G. (1983). The "McDonaldization" of society. Journal of American Culture, 6(1), 100-107.

Ruiz del Olmo, F. (2012). El cine ante las transformaciones y los flujos comunicativos globales. Revista ICONO14. Revista Cientifica de Comunicación y Tecnologías Emergentes, 10(1), 1-7. doi:http://dx.doi.org/10.7195/ri14.v10i1.486

Saffari, B., Maertens, G., \& Miazzo, V. (2010). Patente n ${ }^{\circ}$ US20100312596 A1. Estados Unidos. 
Secretaría de Estado para la Función Pública. (2014). Taxonomía del Catálogo datos.gob.es. Documento de trabajo. Recuperado el 17 de 01 de 2016, de datos.gob.es. Disponible en: http://goo.gl/s3q0El [Fecha de consulta: 2 de febrero de 2016].

SIELOCAL. (2012). Sielocal. Transparencia económica. Disponible en: http://www.sielocal.com/ [Fecha de consulta: 16 de enero de 2016].

Simon, H. A. (1971). Designing Organizations for an Information-Rich World. En M. Greenberger, Computers, Communication, and the Public Interest. Baltimore: The Johns Hopkins Press.

Simón, L. R., Avilés, R. A., Botezan, I., Serrano, S. C., Jiménez, A. S., Jiménez, R. S., \& Gastaminza, F. (2012). De la reutilización de la información del sector público a los portales de datos abiertos. Europa. BiD: textos universitaris de biblioteconomia i documentació, 29.

Symantec. (2014). Proyecto "Honey Stick" sobre smartphones. Edición México. México DF: Symantec.

Symantec. (2012). The Symantec Smartphone Honey Stick Project. Disponible en: https://goo.gl/HpwM3U [Fecha de consulta: 15 de enero de 2016].

The Climate Group. (2014). Urban Mobility in the Smart City Age. Paris: Schneider Electric.

Thomas, D., Beresford, A., \& Rice, A. (2015). Scurity Metrics for the Android Ecosystem. SPSM '15 Proceedings of the 5 th Annual ACM CCS Workshop on Security and Privacy in Smartphones and Mobile Devices, 87-98.

Trejo, R. (2006). Viviendo en el Aleph: la sociedad de la información y sus laberintos. Barcelona: Gedisa.

Usher, N. (2012). Service Journalism as Community experience: Personal technology and personal finance at The New York Times. Journalism Practice, 107-121.

Yousuf Ali, M., \& Kaur, K. (2015). Journalism 2.0: Journalist View about Media Information Service Trends in Karachi, Pakistan. Chinese Librarianship (39).

\footnotetext{
${ }^{\mathrm{i}}$ Agradecimientos: Este estudio está financiado por el proyecto Ciudadanía Digital y Open Data Access: empoderamiento ciudadano a través de los medios sociales en el entorno digital (CSO2012-30756, Ministerio de Economía y Competitividad).
} 